\title{
Lattice design and expected performance of the Muon Ionization Cooling Experiment demonstration of ionization cooling
}

M. Bogomilov, ${ }^{1}$ R. Tsenov, ${ }^{1}$ G. Vankova-Kirilova, ${ }^{1}$ Y. Song, ${ }^{2}$ J. Tang, ${ }^{2}$ Z. Li, ${ }^{3}$ R. Bertoni, ${ }^{4}$ M. Bonesini,${ }^{4}$ F. Chignoli, ${ }^{4}$ R. Mazza, ${ }^{4}$ V. Palladino, ${ }^{5}$ A. de Bari, ${ }^{6}$ G. Cecchet,${ }^{6}$ D. Orestano, ${ }^{7}$ L. Tortora,${ }^{7}$ Y. Kuno, ${ }^{8}$ S. Ishimoto, ${ }^{9}$ F. Filthaut,${ }^{10}$ D. Jokovic, ${ }^{11}$ D. Maletic, ${ }^{11}$ M. Savic, ${ }^{11}$ O. M. Hansen, ${ }^{12}$ S. Ramberger, ${ }^{12}$ M. Vretenar, ${ }^{12}$ R. Asfandiyarov, ${ }^{13}$ A. Blondel, ${ }^{13}$ F. Drielsma, ${ }^{13}$ Y. Karadzhov, ${ }^{13}$ G. Charnley, ${ }^{14}$ N. Collomb, ${ }^{14}$ K. Dumbell, ${ }^{14}$ A. Gallagher, ${ }^{14}$ A. Grant, ${ }^{14}$ S. Griffiths, ${ }^{14}$ T. Hartnett, ${ }^{14}$ B. Martlew, ${ }^{14}$ A. Moss,${ }^{14}$ A. Muir, ${ }^{14}$ I. Mullacrane, ${ }^{14}$ A. Oates,${ }^{14}$ P. Owens, ${ }^{14}$ G. Stokes, ${ }^{14}$ P. Warburton, ${ }^{14}$ C. White, ${ }^{14}$ D. Adams, ${ }^{15}$ R. J. Anderson, ${ }^{15}$ P. Barclay, ${ }^{15}$ V. Bayliss, ${ }^{15}$ J. Boehm, ${ }^{15}$ T. W. Bradshaw, ${ }^{15}$ M. Courthold, ${ }^{15}$ V. Francis, ${ }^{15}$ L. Fry, ${ }^{15}$ T. Hayler, ${ }^{15}$ M. Hills, ${ }^{15}$ A. Lintern, ${ }^{15}$ C. Macwaters ${ }^{15}$ A. Nichols, ${ }^{15}$ R. Preece, ${ }^{15}$ S. Ricciardi, ${ }^{15}$ C. Rogers ${ }^{15}$ T. Stanley, ${ }^{15}$ J. Tarrant, ${ }^{15}$ M. Tucker, ${ }^{15}$ A. Wilson, ${ }^{15}$ S. Watson, ${ }^{16}$ R. Bayes,${ }^{17}$ J. C. Nugent, ${ }^{17}$ F. J. P. Soler, ${ }^{17}$ R. Gamet, ${ }^{18}$ G. Barber, ${ }^{19}$ V. J. Blackmore, ${ }^{19}$ D. Colling, ${ }^{19}$ A. Dobbs, ${ }^{19}$ P. Dornan, ${ }^{19}$ C. Hunt ${ }^{19}$ A. Kurup,${ }^{19}$ J.-B. Lagrange,${ }^{19,}{ }^{*}$ K. Long, ${ }^{19}$ J. Martyniak,${ }^{19}$ S. Middleton, ${ }^{19}$ J. Pasternak, ${ }^{19}$ M. A. Uchida, ${ }^{19}$ J. H. Cobb, ${ }^{20}$ W. Lau, ${ }^{20}$ C. N. Booth, ${ }^{21}$ P. Hodgson, ${ }^{21}$ J. Langlands, ${ }^{21}$ E. Overton, ${ }^{21}$ M. Robinson, ${ }^{21}$ P. J. Smith, ${ }^{21}$ S. Wilbur, ${ }^{21}$ A. J. Dick, ${ }^{22}$ K. Ronald, ${ }^{22}$ C. G. Whyte, ${ }^{22}$ A. R. Young, ${ }^{22}$ S. Boyd ${ }^{23}$ P. Franchini, ${ }^{23}$ J. R. Greis, ${ }^{23}$ C. Pidcott, ${ }^{23}$ I. Taylor, ${ }^{23}$ R. B. S. Gardener ${ }^{24}$ P. Kyberd, ${ }^{24}$ J. J. Nebrensky, ${ }^{24}$ M. Palmer, ${ }^{25}$ H. Witte, ${ }^{25}$ A. D. Bross, ${ }^{26}$ D. Bowring, ${ }^{26}$ A. Liu, ${ }^{26}$ D. Neuffer, ${ }^{26}$ M. Popovic, ${ }^{26}$ P. Rubinov, ${ }^{26}$ A. DeMello, ${ }^{27}$ S. Gourlay, ${ }^{27}$ D. Li, ${ }^{27}$ S. Prestemon, ${ }^{27}$ S. Virostek, ${ }^{27}$ B. Freemire, ${ }^{28}$ P. Hanlet, ${ }^{28}$ D. M. Kaplan, ${ }^{28}$ T. A. Mohayai, ${ }^{28}$ D. Rajaram ${ }^{28}$ P. Snopok, ${ }^{28}$ V. Suezaki, ${ }^{28}$ Y. Torun, ${ }^{28}$ Y. Onel,,${ }^{29}$ L. M. Cremaldi, ${ }^{30}$ D. A. Sanders, ${ }^{30}$ D. J. Summers, ${ }^{30}$ G. G. Hanson, ${ }^{31}$ and C. Heidt ${ }^{31}$

(The MICE collaboration)

${ }^{1}$ Department of Atomic Physics, St. Kliment Ohridski University of Sofia, Sofia 1164, Bulgaria

${ }^{2}$ Institute of High Energy Physics, Chinese Academy of Sciences, Beijing 100039, China

${ }^{3}$ Sichuan University, Sichuan Sheng 610000, China

${ }^{4}$ Sezione INFN Milano Bicocca, Dipartimento di Fisica G. Occhialini, Milano 20126, Italy

${ }^{5}$ Sezione INFN Napoli and Dipartimento di Fisica, Università Federico II,

Complesso Universitario di Monte S. Angelo, Napoli 80126, Italy

${ }^{6}$ Sezione INFN Pavia and Dipartimento di Fisica, Pavia 27100, Italy

${ }^{7}$ INFN Sezione di Roma Tre and Dipartimento di Matematica e Fisica,

Università Roma Tre, 00146 Roma, Italy

${ }^{8}$ Osaka University, Graduate School of Science, Department of Physics,

Toyonaka, Osaka 565-0871, Japan

${ }^{9}$ High Energy Accelerator Research Organization (KEK), Institute of Particle and Nuclear Studies,

Tsukuba 305-0801, Ibaraki, Japan

${ }^{10}$ Nikhef, Amsterdam, The Netherlands and Radboud University, Nijmegen 1098, The Netherlands

${ }^{11}$ Institute of Physics, University of Belgrade, Belgrade 11080, Serbia

${ }^{12}$ CERN, Geneva 1217, Switzerland

${ }^{13}$ DPNC, Section de Physique, Université de Genève, Geneva 1205, Switzerland

${ }^{14}$ STFC Daresbury Laboratory, Daresbury, Cheshire WA4 4AD, United Kingdom

${ }^{15}$ STFC Rutherford Appleton Laboratory, Harwell Oxford, Didcot OX11 OQX, United Kingdom

${ }^{16}$ STFC Rutherford UK Astronomy Technology Centre, Royal Observatory,

Edinburgh, Blackford Hill, Edinburgh EH9 3HJ, United Kingdom

${ }^{17}$ School of Physics and Astronomy, Kelvin Building, The University of Glasgow, Glasgow G12 8SU, United Kingdom

${ }^{18}$ Department of Physics, University of Liverpool, Liverpool L69 7ZE, United Kingdom

${ }^{19}$ Department of Physics, Blackett Laboratory, Imperial College London, London SW7 2BB, United Kingdom

${ }^{20}$ Department of Physics, University of Oxford, Denys Wilkinson Building, Oxford OX1 3PJ, United Kingdom

${ }^{21}$ Department of Physics and Astronomy, University of Sheffield, Sheffield S10 2TN, United Kingdom

${ }^{22}$ SUPA and the Department of Physics, University of Strathclyde, Glasgow G1 1XQ, United Kingdom and Cockroft Institute, United Kingdom

${ }^{23}$ Department of Physics, University of Warwick, Coventry CV4 7AL, United Kingdom

${ }^{24}$ Brunel University, Uxbridge UB8 $3 P H$, United Kingdom

${ }^{25}$ Brookhaven National Laboratory, New York NY 11967, USA

${ }^{26}$ Fermilab, Batavia, Illinois 60510, USA 


\author{
${ }^{27}$ Lawrence Berkeley National Laboratory, Berkeley, California 94720, USA \\ ${ }^{28}$ Illinois Institute of Technology, Chicago, Illinois 60616, USA \\ ${ }^{29}$ Department of Physics and Astronomy, University of Iowa, Iowa City, Iowa 52242, USA \\ ${ }^{30}$ University of Mississippi, Oxford, Mississippi 38677, USA \\ ${ }^{31}$ University of California, Riverside, California 92521, USA \\ (Received 30 January 2017; published 19 June 2017)
}

\begin{abstract}
Muon beams of low emittance provide the basis for the intense, well-characterized neutrino beams necessary to elucidate the physics of flavor at a neutrino factory and to provide lepton-antilepton collisions at energies of up to several $\mathrm{TeV}$ at a muon collider. The international Muon Ionization Cooling Experiment (MICE) aims to demonstrate ionization cooling, the technique by which it is proposed to reduce the phasespace volume occupied by the muon beam at such facilities. In an ionization-cooling channel, the muon beam passes through a material in which it loses energy. The energy lost is then replaced using rf cavities. The combined effect of energy loss and reacceleration is to reduce the transverse emittance of the beam (transverse cooling). A major revision of the scope of the project was carried out over the summer of 2014. The revised experiment can deliver a demonstration of ionization cooling. The design of the cooling demonstration experiment will be described together with its predicted cooling performance.
\end{abstract}

DOI: 10.1103/PhysRevAccelBeams.20.063501

\section{INTRODUCTION}

Stored muon beams have been proposed as the source of neutrinos at a neutrino factory $[1,2]$ and as the means to deliver multi-TeV lepton-antilepton collisions at a muon collider [3,4]. In such facilities the muon beam is produced from the decay of pions generated by a high-power proton beam striking a target. The tertiary muon beam occupies a large volume in phase space. To optimize the muon yield while maintaining a suitably small aperture in the muonacceleration system requires that the muon beam be "cooled" (i.e., its phase-space volume reduced) prior to acceleration. A muon is short-lived, decaying with a lifetime of $2.2 \mu \mathrm{s}$ in its rest frame. Therefore, beam manipulation at low energy $(\leq 1 \mathrm{GeV})$ must be carried out rapidly. Four cooling techniques are in use at particle accelerators: synchrotron-radiation cooling [5]; laser cooling [6-8]; stochastic cooling [9]; and electron cooling [10]. Synchrotron-radiation cooling is observed only in electron or positron beams, owing to the relatively low mass of the electron. Laser cooling is limited to certain ions and atomic beams. Stochastic cooling times are dependent on the bandwidth of the stochastic-cooling system relative to the frequency spread of the particle beam. The electron-cooling time is limited by the available electron density and the electron-beam energy and emittance. Typical cooling times are between seconds and hours, long compared with the muon lifetime. Ionization cooling proceeds by passing a muon beam through a material, the absorber, in which it

j.lagrange@imperial.ac.uk

Published by the American Physical Society under the terms of the Creative Commons Attribution 4.0 International license. Further distribution of this work must maintain attribution to the author(s) and the published article's title, journal citation, and DOI. loses energy through ionization, and subsequently restoring the lost energy in accelerating cavities. Transverse and longitudinal momentum are lost in equal proportions in the absorber, while the cavities restore only the momentum component parallel to the beam axis. The net effect of the energy-loss/reacceleration process is to decrease the ratio of transverse to longitudinal momentum, thereby decreasing the transverse emittance of the beam. In an ionizationcooling channel the cooling time is short enough to allow the muon beam to be cooled efficiently with modest decay losses. Ionization cooling is therefore the technique by which it is proposed to cool muon beams [11-13]. This technique has never been demonstrated experimentally and such a demonstration is essential for the development of future high-brightness muon accelerators.

The international Muon Ionization Cooling Experiment (MICE) collaboration proposes a two-part process to perform a full demonstration of transverse ionization cooling. First, the "Step IV" configuration [14] will be used to study the material and beam properties that determine the performance of an ionization-cooling lattice. Second, a study of transverse-emittance reduction in a cooling cell that includes accelerating cavities will be performed.

The cooling performance of an ionization-cooling cell depends on the emittance and momentum of the initial beam, on the properties of the absorber material and on the transverse betatron function $\left(\beta_{\perp}\right)$ at the absorber. These factors will be studied using the Step IV configuration. Once this has been done, "sustainable" ionization cooling must be demonstrated. This requires restoring energy lost by the muons as they pass through the absorber using $\mathrm{rf}$ cavities. The experimental configuration with which the MICE collaboration originally proposed to study ionization cooling was presented in [15]. This configuration was revised to accelerate the timetable on which a demonstration of ionization cooling could be delivered and to reduce 
cost. This paper describes the revised lattice proposed by the MICE collaboration for the demonstration of ionization cooling and presents its performance.

\section{COOLING IN NEUTRINO FACTORIES AND MUON COLLIDERS}

At production, muons occupy a large volume of phase space. The emittance of the initial muon beam must be reduced before the beam is accelerated. A neutrino factory [16] requires the transverse emittance to be reduced from $15-20 \mathrm{~mm}$ to $2-5 \mathrm{~mm}$. A muon collider [17] requires the muon beam to be cooled in all six phase-space dimensions; to achieve the desired luminosity requires an emittance of $\sim 0.025 \mathrm{~mm}$ in the transverse plane and $\sim 70 \mathrm{~mm}$ in the longitudinal direction $[18,19]$.

Ionization cooling is achieved by passing a muon beam through a material with low atomic number $(Z)$, in which it loses energy by ionization, and subsequently accelerating the beam. The rate of change of the normalized transverse emittance, $\varepsilon_{\perp}$, is given approximately by $[12,20,21]$ :

$$
\frac{\mathrm{d} \varepsilon_{\perp}}{\mathrm{d} z} \simeq-\frac{\varepsilon_{\perp}}{\beta^{2} E_{\mu}}\left\langle\frac{\mathrm{d} E}{\mathrm{~d} z}\right\rangle+\frac{\beta_{\perp}(13.6 \mathrm{MeV} / c)^{2}}{2 \beta^{3} E_{\mu} m_{\mu} X_{0}} ;
$$

where $z$ is the longitudinal coordinate, $\beta c$ is the muon velocity, $E_{\mu}$ the energy, $\left\langle\frac{\mathrm{d} E}{\mathrm{~d} z}\right\rangle$ the mean rate of energy loss per unit path-length, $m_{\mu}$ the mass of the muon, $X_{0}$ the radiation length of the absorber and $\beta_{\perp}$ the transverse betatron function at the absorber. The first term of this equation describes "cooling" by ionization energy loss and the second describes "heating" by multiple Coulomb scattering. Equation (1) implies that the equilibrium emittance, for which $\frac{d \varepsilon_{\perp}}{d z}=0$, and the asymptotic value of $\frac{d \varepsilon_{\perp}}{d z}$ for large emittance are functions of muon-beam energy.

In order to have good performance in an ionizationcooling channel, $\beta_{\perp}$ needs to be minimized and $X_{0}\left\langle\frac{\mathrm{d} E}{\mathrm{~d} z}\right\rangle$ maximised. The betatron function at the absorber is minimized using a suitable magnetic focusing channel (typically solenoidal) [22,23] and $X_{0}\left\langle\frac{\mathrm{d} E}{\mathrm{~d} z}\right\rangle$ is maximized using a low- $Z$ absorber such as liquid hydrogen $\left(\mathrm{LH}_{2}\right)$ or lithium hydride $(\mathrm{LiH})$ [24].

\section{THE MUON IONIZATION COOLING EXPERIMENT}

The muons for MICE come from the decay of pions produced at an internal target dipping directly into the circulating proton beam in the ISIS synchrotron at the Rutherford Appleton Laboratory (RAL) $[25,26]$. A beam line of 9 quadrupoles, 2 dipoles and a superconducting "decay solenoid" collects and transports the momentumselected beam into the experiment [27]. The small fraction of pions that remain in the beam may be rejected during analysis using the time-of-flight hodoscopes and Cherenkov counters that are installed in the beam line upstream of the experiment [28]. A diffuser is installed at the upstream end of the experiment to vary the initial emittance of the beam. Ionization cooling depends on momentum through $\beta, E_{\mu}$ and $\left\langle\frac{\mathrm{d} E}{\mathrm{~d} z}\right\rangle$ as shown in Eq. (1). It is therefore proposed that the performance of the cell be measured for momenta in the range $140 \mathrm{MeV} / \mathrm{c}$ to $240 \mathrm{MeV} / c$ [15].

\section{A. The configuration of the ionization-cooling experiment}

The configuration proposed for the demonstration of ionization cooling is shown in Fig. 1. It contains a cooling cell sandwiched between two spectrometer-solenoid modules. The cooling cell is composed of two $201 \mathrm{MHz}$ cavities, one primary $(65 \mathrm{~mm})$ and two secondary $(32.5 \mathrm{~mm}) \mathrm{LiH}$ absorbers placed between two superconducting "focus-coil" (FC) modules. Each FC has two separate windings that can be operated either with the same or in opposed polarity.

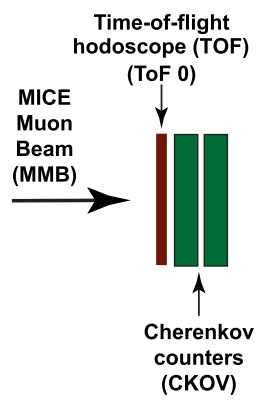

MICE

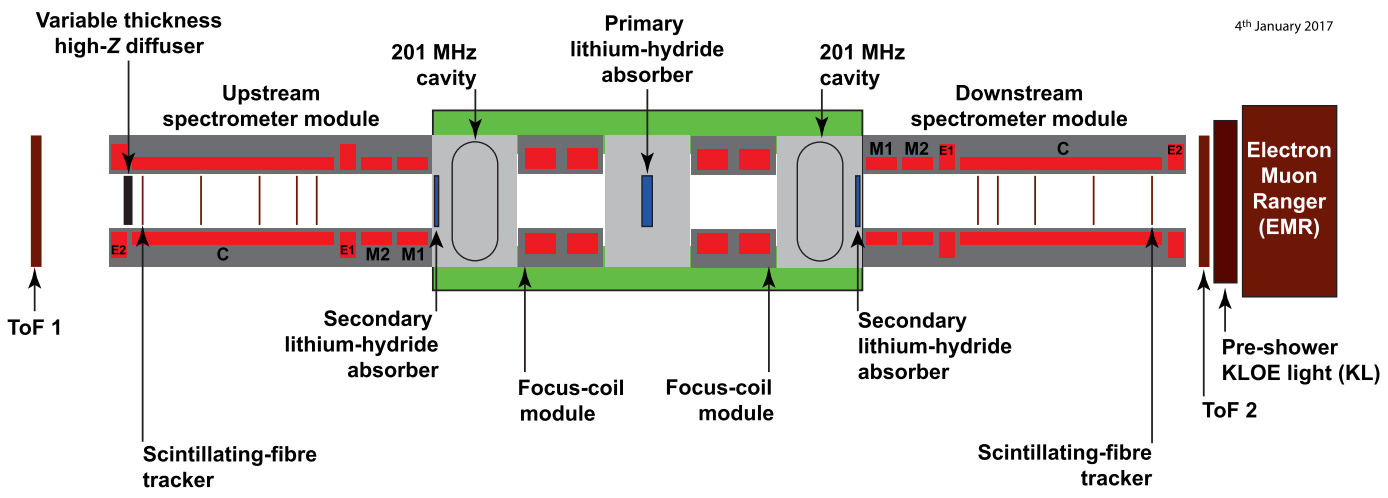

FIG. 1. Layout of the lattice configuration for the cooling demonstration. The red rectangles represent the solenoids. The individual coils in the spectrometer solenoids are labeled E1, C, E2, M1 and M2. The ovals represent the rf cavities and the blue rectangles the absorbers. The various detectors (time-of-flight hodoscopes [29,30], Cerenkov counters [31], scintillating-fibre trackers [32], KLOE Light (KL) calorimeter [27,33], electron muon ranger [34]) used to characterize the beam are also represented. The green-shaded box indicates the cooling cell. 
The emittance is measured upstream and downstream of the cooling cell using scintillating-fiber tracking detectors [32] immersed in the uniform $4 \mathrm{~T}$ magnetic field provided by three superconducting coils (E1, C, E2). The trackers are used to reconstruct the trajectories of individual muons at the entrance and exit of the cooling cell. The reconstructed tracks are combined with information from instrumentation upstream and downstream of the spectrometer modules to measure the muon-beam emittance at the upstream and downstream tracker reference planes. The instrumentation upstream and downstream of the spectrometer modules serves to select a pure sample of muons. Time-of-flight hodoscopes are used to determine the time at which the muon crosses the rf cavities. The spectrometer-solenoid magnets also contain two superconducting "matching" coils (M1, M2) that are used to match the optics between the uniform field region and the neighboring FC.

The secondary $\mathrm{LiH}$ absorbers (SAs) are introduced between the cavities and the trackers to minimize the exposure of the trackers to "dark-current" electrons originating from the rf cavities. Experiments at the MuCool Test Area (MTA) at Fermilab [35] have observed that the rate of direct $\mathrm{X}$-ray production from the rf cavities can be managed to ensure it does not damage the trackers [36]. The SAs are introduced to minimize the exposure of the trackers to energetic dark-current electrons that could produce background hits. The SAs are positioned between the trackers and the cavities such that they can be removed to study the empty channel. The SAs increase the net transverse-cooling effect since the betatron functions at these locations are small.

Retractable lead radiation shutters will be installed on rails between the spectrometer solenoids and the rf modules to protect the trackers against dark-current induced radiation during cavity conditioning. The SAs will be mounted on a rail system similar to that which will be used for the lead shutters and will be located between the cavities and the lead shutters. Both mechanisms will be moved using linear piezoelectric motors that operate in vacuum and magnetic field. The design of both the radiation shutter and the movable SA inside the vacuum chamber is shown in Fig. 2.

The rf cavities are $201 \mathrm{MHz}$ "pillbox" resonators, $430 \mathrm{~mm}$ in length, operating in the $\mathrm{TM}_{010}$ mode with large diameter apertures to accommodate the high emittance beam. The apertures are covered by thin $(0.38 \mathrm{~mm})$ beryllium windows to define the limits for the accelerating rf fields whilst minimizing the scattering of muons. The cavity is excited by two magnetic-loop couplers on opposite sides of the cavity. At the particle rate expected in MICE there is no beam-loading of the rf fields. An effective peak field of $10.3 \mathrm{MV} / \mathrm{m}$ is expected for a drive power of 1.6 MW to each cavity. This estimate was used to define the gradient in the simulations described below.

The original configuration of the MICE cooling cell described in [15] was composed of three focus-coil modules, each of which housed a liquid-hydrogen absorber, and two,

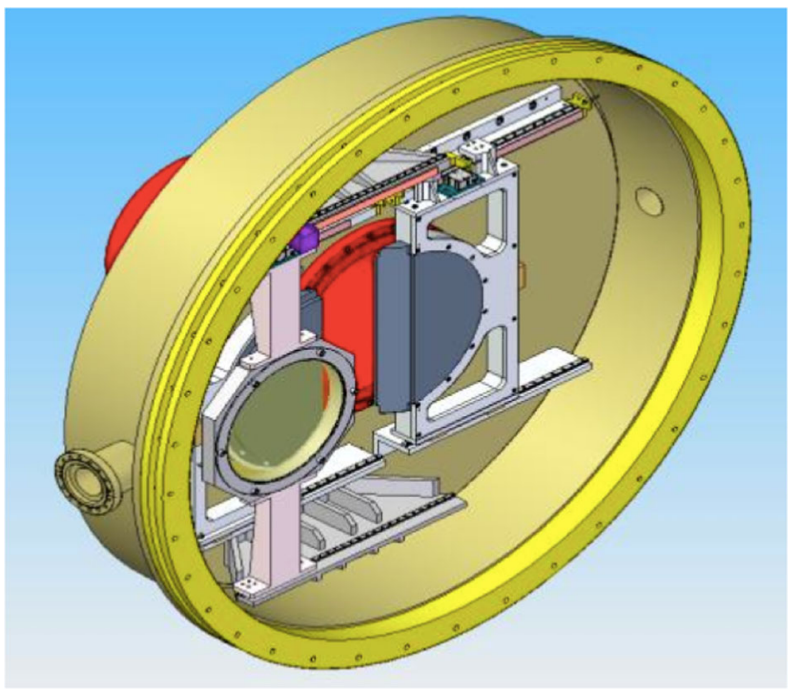

FIG. 2. Design of the movable frame for the secondary absorber (front) and the lead radiation shutter (back). The half discs of the lead shutter (grey) can be seen together with the rails (white) inside the vacuum chamber (yellow).

four-cavity, linac modules. Each linac module incorporated a large, superconducting "coupling coil" to transport the beam. The configuration described in this paper was developed to simplify the lattice described in [15] such that the coupling coils are not required and acceleration is provided by two single-cavity modules. The revision of the magnetic lattice substantially reduces the technical risks associated with the implementation of the experiment since all of the superconducting solenoids required to transport and focus the beam have been commissioned on the beam line. Further, by reducing the number of cavities from eight to two and reconfiguring the rf-power-distribution system the cost of implementing the experiment has been reduced and the timetable on which the experiment can be mounted has been advanced. The present configuration was optimized to maximize its cooling performance as described in Sec. IV. The performance of the optimized lattice, though reduced compared to that described in [15], is sufficient for the principle of ionization-cooling to be demonstrated (see Sec. VI).

\section{LATTICE DESIGN}

\section{A. Design parameters}

The lattice has been optimized to maximize the reduction in transverse emittance. The optimum is obtained by matching the betatron function to a small value in the central absorber while minimizing its maximum values in the FC modules; limiting the size of the betatron function in the FCs helps to reduce the influence of nonlinear terms in the magnetic-field expansion. The matching accounts for the change in energy of the muons as they pass through the cooling cell by adjusting currents in the upstream and 
downstream FCs and in the matching coils in the spectrometer solenoids independently while maintaining the field in the tracking volumes at $4 \mathrm{~T}$. In this configuration, it is also possible to keep the betatron function relatively small at the position of the secondary absorbers whilst maintaining an acceptable beam size at the position of the cavities.

Chromatic aberrations caused by the large momentum spread of the beam ( $5 \% \mathrm{rms})$ lead to a chromatic mismatch of the beam in the downstream solenoid unless the phase advance across the cooling cell (i.e., the rate of rotation of the phase-space ellipse) is chosen appropriately. The phase advance of the cell is obtained by integrating the inverse of the beta-function along the beam axis from the reference plane in the upstream spectrometersolenoid to the reference plane in the downstream spectrometer-solenoid. Such a mismatch reduces the effective transverse-emittance reduction through the chromatic decoherence that results from the superposition of beam evolutions for the different betatron frequencies that result from the range of momenta in the beam. For beams with a large input emittance, spherical aberrations may lead to phase-space filamentation. The chromatic and spherical aberrations were studied by tracking samples of muons through the lattice using the "MICE Analysis User Software" (MAUS, see Sec. V). The betatron-function and emittance evolution of a $200 \mathrm{MeV} / c$ beam with the

TABLE I. General parameters of the initial beam conditions used in the simulations.

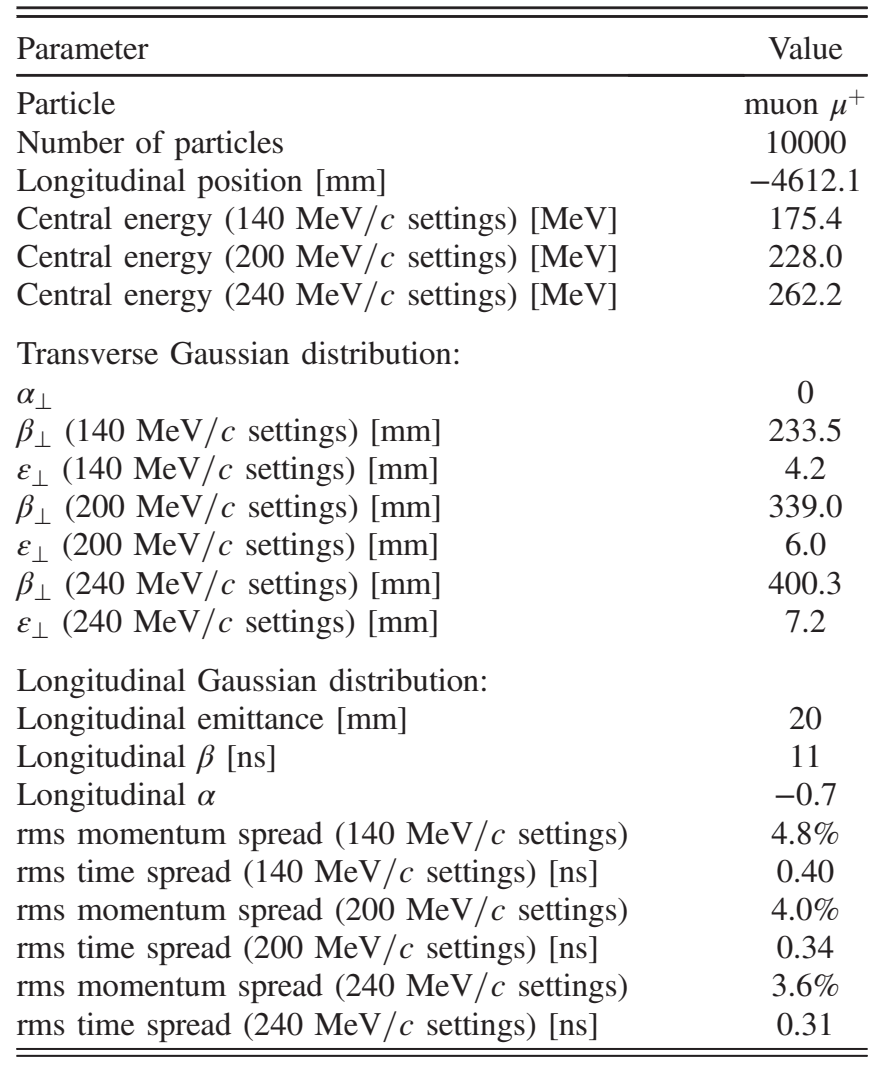

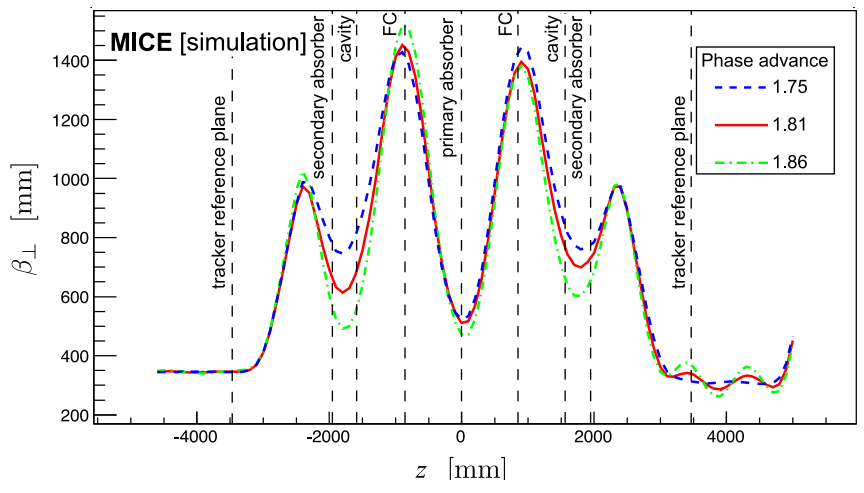

FIG. 3. Transverse 4D beta-function versus longitudinal coordinate $z$ in the cooling-demonstration lattice for $200 \mathrm{MeV} / c$ settings with a phase advance of $2 \pi \times 1.75$ (dashed blue line), $2 \pi \times 1.81$ (solid red line) and $2 \pi \times 1.86$ (dot-dashed green line). The vertical dashed lines with labels show the positions of the tracker reference planes and the centers of the absorbers, rf cavities, and focus coil modules.

initial parameters given in Table I are shown, for different phase advances, in Figs. 3 and 4, respectively. The phase advance of $2 \pi \times 1.81$ showed the largest transverseemittance reduction and was therefore chosen. The lattice parameters for this phase advance are presented in Table II.

The currents that produce the optimum magnetic lattice were obtained using the procedure described above for three momentum settings: $140 \mathrm{MeV} / c, 200 \mathrm{MeV} / c$, and $240 \mathrm{MeV} / c$. The magnetic field on axis for each of these settings is shown in Fig. 5. The fields in the downstream FC and spectrometer are opposite to those in the upstream FC and spectrometer, the field changing sign at the primary absorber. Such a field flip is required in an ionization cooling channel to reduce the build-up of canonical angular momentum [37]. The currents required to produce the magnetic fields shown in Fig. 5 are listed in Table III. All currents are within the proven limits of operation for the

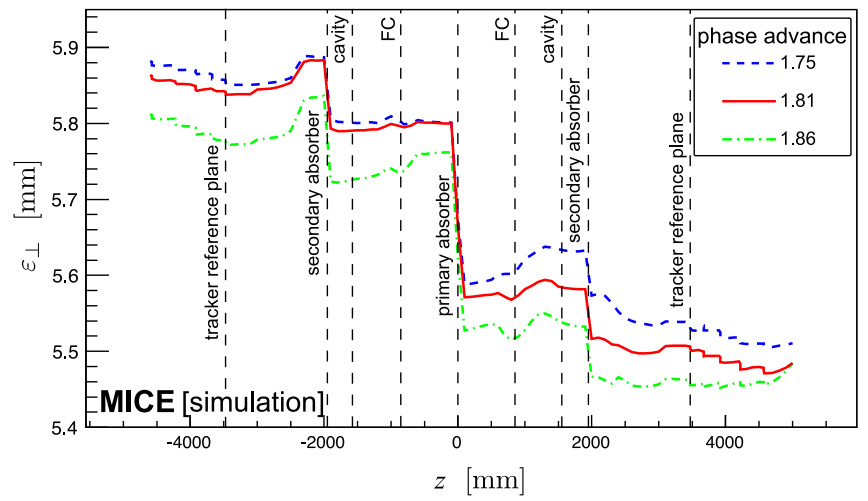

FIG. 4. 4D emittance evolution in the cooling-demonstration lattice for $200 \mathrm{MeV} / c$ settings with a phase advance of $2 \pi \times 1.75$ (dashed blue line), $2 \pi \times 1.81$ (solid red line) and $2 \pi \times 1.86$ (dotdashed green line). The vertical dashed lines with labels show the positions of the tracker reference planes and the centers of the absorbers, rf cavities, and focus coil modules. 
TABLE II. Parameters of the cooling-demonstration lattice. $L_{\mathrm{SS} \rightarrow \mathrm{FC}}$ is the distance between the center of the spectrometer solenoid and the center of the neighboring $\mathrm{FC}, L_{\mathrm{FC} \rightarrow \mathrm{FC}}$ the distance between the centers of the FCs, and $L_{\mathrm{RF} \text { module } \rightarrow \mathrm{FC}}$ the distance between the rf module and the neighboring FC.

\begin{tabular}{lc}
\hline \hline Parameter & Value \\
\hline Length $L_{\mathrm{SS} \rightarrow \mathrm{FC}}[\mathrm{mm}]$ & 2607.5 \\
Length $L_{\mathrm{FC} \rightarrow \mathrm{FC}}[\mathrm{mm}]$ & 1678.8 \\
Length $L_{\mathrm{rf} \text { module } \rightarrow \mathrm{FC}}[\mathrm{mm}]$ & 784.0 \\
rf Gradient $[\mathrm{MV} / \mathrm{m}]$ & 10.3 \\
Number of rf cavities & 2 \\
Number of primary absorbers & 1 \\
Number of secondary absorbers & 2 \\
\hline \hline
\end{tabular}

individual coil windings. The magnetic forces acting on the coils have been analyzed and were found to be acceptable. Configurations in which there is no field flip can also be considered.

Figure 6 shows matched betatron functions versus longitudinal position for beams of different initial momentum. These betatron functions are constrained, within the fiducial-volume of the trackers, by the requirements on the Courant-Snyder parameters $\alpha_{\perp}=0$ and $\beta_{\perp}=\frac{2 p_{z}}{e B_{z}}$ (where $p_{z}$ is the mean longitudinal momentum of the beam, $e$ the elementary charge and $B_{z}$ the longitudinal component of the magnetic field). A small betatron-function "waist" in the central absorber is achieved. Betatron-function values at relevant positions in the different configurations are summarized in Table IV.

\section{SIMULATION}

Simulations to evaluate the performance of the lattice have been performed using the official MICE simulation

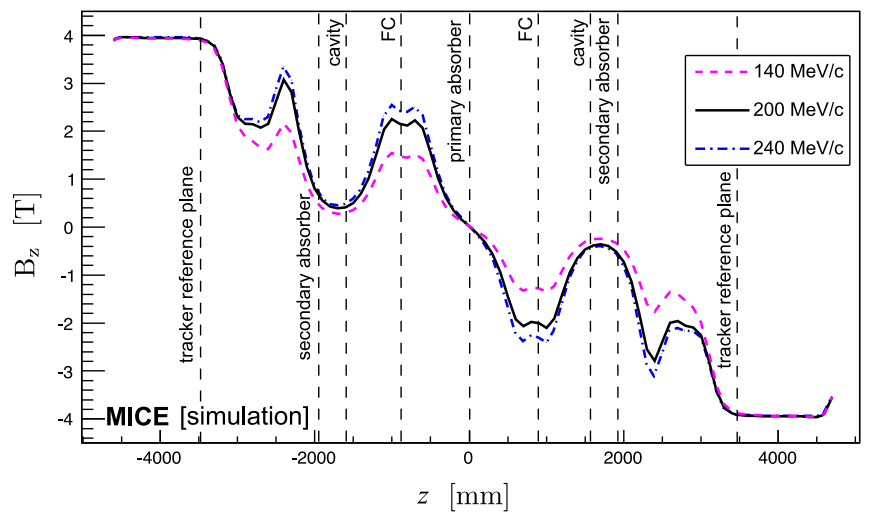

FIG. 5. Magnetic field $B_{z}$ on-axis versus the longitudinal coordinate $z$ for the cooling-demonstration lattice design for $200 \mathrm{MeV} / c$ (solid black line), $140 \mathrm{MeV} / c$ (dashed purple line), and $240 \mathrm{MeV} / c$ (dot-dashed blue line) settings. The vertical dashed lines with labels show the positions of the tracker reference planes and the centres of the absorbers, rf cavities, and focus coil modules.
TABLE III. Coil currents used for $140 \mathrm{MeV} / c, 200 \mathrm{MeV} / c$, and $240 \mathrm{MeV} / c$ lattice settings.

\begin{tabular}{lccc}
\hline \hline & $140 \mathrm{MeV} / c$ & $200 \mathrm{MeV} / c$ & $240 \mathrm{MeV} / c$ \\
Coil & Lattice [A] & Lattice [A] & Lattice [A] \\
\hline Upstream E2 & +253.00 & +253.00 & +253.00 \\
Upstream C & +274.00 & +274.00 & +274.00 \\
Upstream E1 & +234.00 & +234.00 & +234.00 \\
Upstream M2 & +126.48 & +155.37 & +163.50 \\
Upstream M1 & +175.89 & +258.42 & +280.72 \\
Upstream & +54.14 & +79.35 & +89.77 \\
$\quad$ FC-coil 1 & & & \\
Upstream & +54.14 & +79.35 & +89.77 \\
$\quad$ FC-coil 2 & & & \\
Downstream & -47.32 & -74.10 & -85.35 \\
$\quad$ FC-coil 1 & & & \\
Downstream & -47.32 & -74.10 & -85.35 \\
$\quad$ FC-coil 2 & & & \\
Downstream M1 & -140.43 & -231.60 & -261.71 \\
Downstream M2 & -100.12 & -149.15 & -159.21 \\
Downstream E1 & -234.00 & -234.00 & -234.00 \\
Downstream C & -274.00 & -274.00 & -274.00 \\
Downstream E2 & -253.00 & -253.00 & -253.00 \\
\hline \hline
\end{tabular}

and reconstruction software MAUS (MICE Analysis User Software) [38]. In addition to simulation, MAUS also provides a framework for data analysis. MAUS is used for offline analysis and to provide fast real-time detector reconstruction and data visualisation during MICE running. MAUS uses GEANT4 [39,40] for beam propagation and the simulation of detector response. ROOT [41] is used for data visualisation and for data storage.

Particle tracking has been performed for several configurations. The parameters of the initial beam configurations used for the simulations are summarized in Table I.

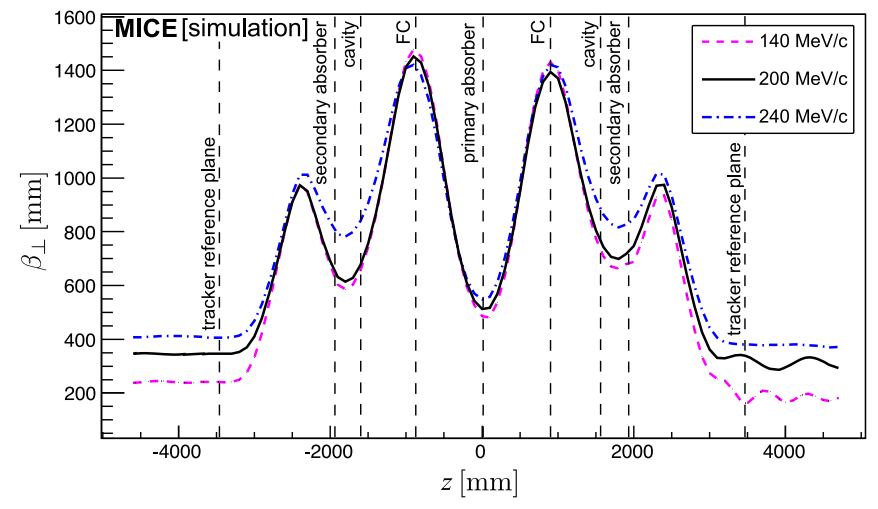

FIG. 6. $\quad \beta_{\perp}$ versus the longitudinal coordinate $z$ for $200 \mathrm{MeV} / c$ (solid black line), $140 \mathrm{MeV} / c$ (dashed purple line) and $240 \mathrm{MeV} / c$ (dot-dashed blue line) in the cooling-demonstration lattice. The vertical dashed lines with labels show the positions of the tracker reference planes and the centers of the absorbers, $\mathrm{rf}$ cavities, and focus coil modules. 
TABLE IV. Beta-function values at relevant positions for an initial beam at $140 \mathrm{MeV} / c, 200 \mathrm{MeV} / c$, and $240 \mathrm{MeV} / c$ in the cooling-demonstration lattice design.

\begin{tabular}{|c|c|c|c|}
\hline Parameter & $\begin{array}{c}\text { Value for } \\
140 \mathrm{MeV} / c\end{array}$ & $\begin{array}{c}\text { Value for } \\
200 \mathrm{MeV} / c\end{array}$ & $\begin{array}{c}\text { Value for } \\
240 \mathrm{MeV} / c\end{array}$ \\
\hline $\begin{array}{l}\beta_{\perp} \text { at primary } \\
\text { absorber }[\mathrm{mm}]\end{array}$ & 480 & 512 & 545 \\
\hline $\begin{array}{l}\beta_{\perp} \text { at upstream } \\
\text { secondary } \\
\text { absorber }[\mathrm{mm}]\end{array}$ & 660 & 710 & 840 \\
\hline $\begin{array}{l}\beta_{\perp} \text { at downstream } \\
\text { secondary } \\
\text { absorber }[\mathrm{mm}]\end{array}$ & 680 & 740 & 850 \\
\hline$\beta_{\perp \max }$ at $\mathrm{FC}[\mathrm{mm}]$ & 1480 & 1450 & 1430 \\
\hline
\end{tabular}

The simulation of the beam starts at a point between the diffuser and the first plane of the tracker. The beam is generated by a randomizing algorithm with a fixed seed. The number of particles launched for each simulation is a compromise between the statistical uncertainty required $(\approx 1 \%)$ and computing time. Each cavity is simulated by a $\mathrm{TM}_{010}$ ideal cylindrical pillbox with a peak effective gradient matched to that expected for the real cavities. The reference particle is used to set the phase of the cavities so that it is accelerated "on crest." The initial distributions defined in Table I are centred on the reference particle in both time and momentum. Table $\mathrm{V}$ lists the acceptance criteria applied to all analyses presented here. Trajectories that fail to meet the acceptance criteria are removed from the analysis.

The normalized transverse emittance is calculated by taking the fourth root of the determinant of the fourdimensional phase-space covariance matrix $[20,21]$. The MICE collaboration plans to take data such that the statistical uncertainty on the relative change in emittance for a particular setting is $1 \%$. The MICE instrumentation was designed such that the systematic uncertainty related to the reconstruction of particle trajectories would contribute at the $\sim 0.3 \%$ level to the overall systematic uncertainty [15]; such uncertainties would thus be negligible.

\section{PERFORMANCE}

Figure 7 shows the evolution of the mean energy of a muon beam as it traverses the lattice. Beams with initial normalised transverse emittance $\varepsilon_{\perp}=4.2 \mathrm{~mm}$,

TABLE V. Acceptance criteria for analysis.

\begin{tabular}{lc}
\hline \hline Parameter & Acceptance condition \\
\hline Particle & muon $\mu^{+}$ \\
Transmission: pass through two planes & $z=-4600 \mathrm{~mm}$ \\
& and $z=5000 \mathrm{~mm}$ \\
Radius at $z=-4600 \mathrm{~mm}$ & $\leq 150.0 \mathrm{~mm}$ \\
Radius at $z=5000 \mathrm{~mm}$ & $\leq 150.0 \mathrm{~mm}$ \\
\hline \hline
\end{tabular}

$\varepsilon_{\perp}=6 \mathrm{~mm}$, and $\varepsilon_{\perp}=7.2 \mathrm{~mm}$ for initial muon beam momenta of $140 \mathrm{MeV} / c, 200 \mathrm{MeV} / c$, and $240 \mathrm{MeV} / c$ respectively are shown. The initial normalized transverse emittance is chosen such that the geometrical emittance of the three beams is the same. A $200 \mathrm{MeV} / c$ muon passing through two $32.5 \mathrm{~mm}$ thick secondary $\mathrm{LiH}$ absorbers and one $65 \mathrm{~mm}$ thick primary $\mathrm{LiH}$ absorber loses an energy of $18.9 \mathrm{MeV}$. Including losses in the scintillating-fiber
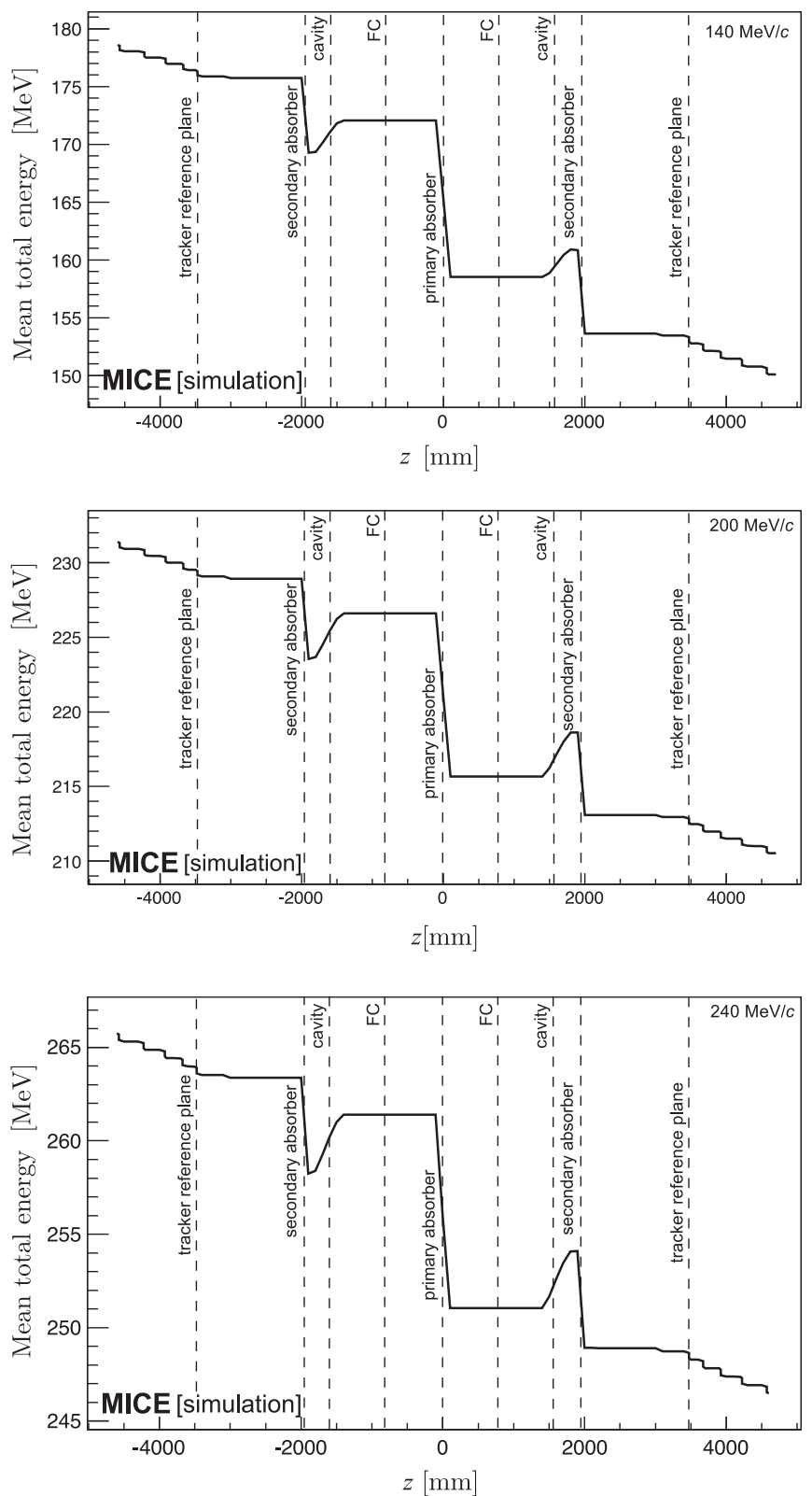

FIG. 7. Mean energy of the beam versus longitudinal coordinate $(z)$ in the cooling-demonstration lattice. Top: the $140 \mathrm{MeV} / c$ configuration for initial emittance $\varepsilon_{\perp}=4.2 \mathrm{~mm}$. Middle: the $200 \mathrm{MeV} / c$ configuration for initial emittance $\varepsilon_{\perp}=6 \mathrm{~mm}$. Bottom: the $240 \mathrm{MeV} / c$ configuration for initial emittance $\varepsilon_{\perp}=7.2 \mathrm{~mm}$. The vertical dashed lines with labels show the positions of the tracker reference planes, and the centers of the absorbers, rf cavities, and focus coil modules. 
trackers and windows, this increases to $24.3 \mathrm{MeV}$. The accelerating gradient that can be achieved in each of the two cavities is constrained by the available rf power and is insufficient to replace all the lost energy. Therefore, a comparison of beam energy with and without acceleration is required. With acceleration an energy
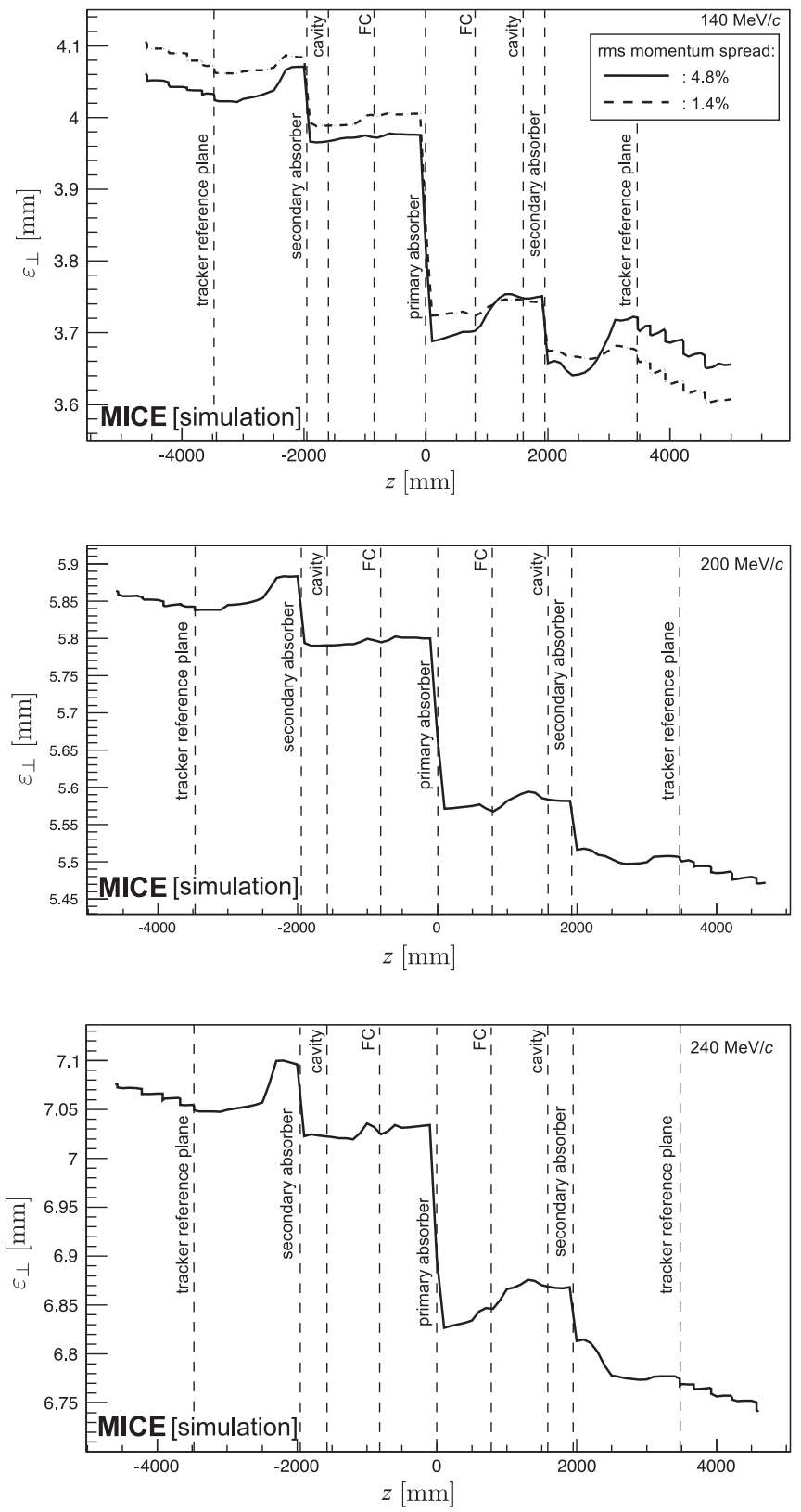

FIG. 8. Emittance variation versus the longitudinal coordinate (z) for the cooling-demonstration lattice design. Top: $140 \mathrm{MeV} / \mathrm{c}$ beam with initial $\varepsilon_{\perp}=4.2 \mathrm{~mm}$ with an rms momentum spread of 6.7 MeV/c (rms spread 4.8\%, solid line) and $2.5 \mathrm{MeV} / c$ (rms spread $1.8 \%$, dashed line). Middle: $200 \mathrm{MeV} / \mathrm{c}$ beam with initial $\varepsilon_{\perp}=6 \mathrm{~mm}$ (rms spread 4.0\%). Bottom: $240 \mathrm{MeV} / \mathrm{c}$ beam with initial $\varepsilon_{\perp}=7.2 \mathrm{~mm}$ (rms spread 3.6\%). The vertical dashed lines with labels show the positions of the tracker reference planes, and the centers of the absorbers, rf cavities, and focus coil modules. deficit of $\langle\Delta E\rangle=19 \mathrm{MeV}$ will be observed. This measurable difference will be used to extrapolate the measured cooling effect to that which would pertain if all the lost energy were restored.

The evolution of normalized transverse emittance across the lattice is shown in Fig. 8. The beam is subject to nonlinear effects in regions of high $\beta_{\perp}$, which cause the normalized transverse emittance to grow, especially in the $140 \mathrm{MeV} / c$ configuration. This phenomenon can be seen in three different regions of the lattice: a moderate increase in emittance is observed at $z \approx-2500 \mathrm{~mm}$ and $z \approx 1000 \mathrm{~mm}$ while a larger increase is observed at $z \approx 3000 \mathrm{~mm}$. The nonlinear effects are mainly chromatic in origin, since they are greatly lessened when the initial momentum spread is reduced. This is illustrated for the $140 \mathrm{MeV} / c$ case for which the evolution of normalized emittance for beams with an rms momentum spread of 6.7 MeV/c and $2.5 \mathrm{MeV} / c$ are shown. Nonetheless, in all cases a reduction in emittance is observed between the upstream and downstream trackers $(z= \pm 3473 \mathrm{~mm})$. The lattice is predicted to achieve an emittance reduction between the tracker reference planes of $\approx 8.1 \%, \approx 5.8 \%$ and $\approx 4.0 \%$ in the $140 \mathrm{MeV} / c, 200 \mathrm{MeV} / c$, and $240 \mathrm{MeV} / c$ cases, respectively. A reduction as large as $\approx 10 \%$ can be reached in the $140 \mathrm{MeV} / c$ configuration with an $\mathrm{rms}$ momentum spread of $1.4 \%$.

The transmission of the cooling-demonstration lattice for beams of mean momentum $140 \mathrm{MeV} / c, 200 \mathrm{MeV} / c$, and $240 \mathrm{MeV} / c$ is shown in Fig. 9. Transmission is computed as the ratio of the number of particles that satisfy the acceptance criteria observed downstream of the cooling cell divided by the number that enter the cell. This accounts

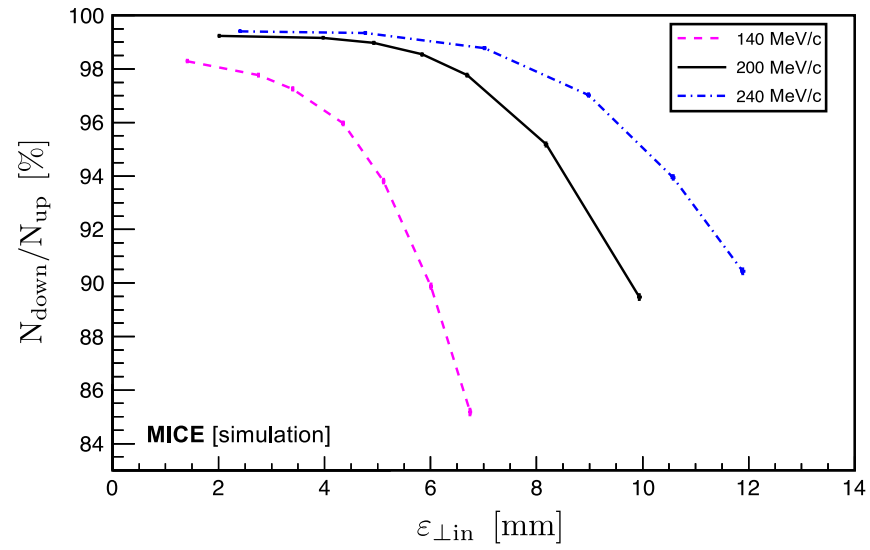

FIG. 9. Transmission (defined as the ratio of good muons observed downstream of the cooling cell, $N_{\text {down }}$, to those observed upstream, $N_{\text {up }}$ ) in percent versus initial emittance $\left(\varepsilon_{\perp \text { in }}\right)$ for the cooling-demonstration lattice. The transmission of the $140 \mathrm{MeV} / c, 200 \mathrm{MeV} / c$, and $240 \mathrm{MeV} / c$ lattices are shown as the purple-dashed, solid black, and dot-dashed blue lines respectively. The error bars indicate the statistical precision that would be achieved using a sample of 100,000 muons. 
for decay losses and implies that, in the absence of scraping or acceptance losses, the maximum transmission for beams of mean momentum $140 \mathrm{MeV} / c, 200 \mathrm{MeV} / c$, and $240 \mathrm{MeV} / c$ is $98.9 \%, 99.2 \%$, and $99.5 \%$, respectively. The lattice delivers transmission close to the maximum for $200 \mathrm{MeV} / c$ and $240 \mathrm{MeV} / c$ beams with input emittance below $\approx 5 \mathrm{~mm}$ and $\approx 7 \mathrm{~mm}$, respectively. For beams of larger input emittance, the transmission gradually decreases with increasing initial emittance due to the scraping of high amplitude muons. The beam is subject to chromatic effects in regions of high $\beta_{\perp}$, which causes nonlinear emittance growth and limits the transmission. The behavior of the transmission for the various beam energies results from the different geometrical emittance values of the beam for the same initial normalised emittance and the energy dependence of the energy loss and scattering in the material through which the beam passes.

The fractional change in normalized transverse emittance with respect to the input emittance for beams of mean momentum $140 \mathrm{MeV} / c, 200 \mathrm{MeV} / c$, and $240 \mathrm{MeV} / c$ is shown in Fig. 10. The different values of the equilibrium emittance and the asymptote at large emittance for each momentum are clearly visible in Fig. 10. A maximum cooling effect of $15 \%, 8 \%$, and $6 \%$ can be observed for beams with $140 \mathrm{MeV} / c, 200 \mathrm{MeV} / c$, and $240 \mathrm{MeV} / c$, respectively.

The performance of the configuration proposed here is comparable to that described in [15]. In the "Step V" configuration, that incorporated two liquid-hydrogen absorbers each placed within a focus-coil module capable of providing a value $\beta_{\perp}$ smaller than that which can be achieved with the present lattice, the maximum cooling effect with an input momentum and emittance of

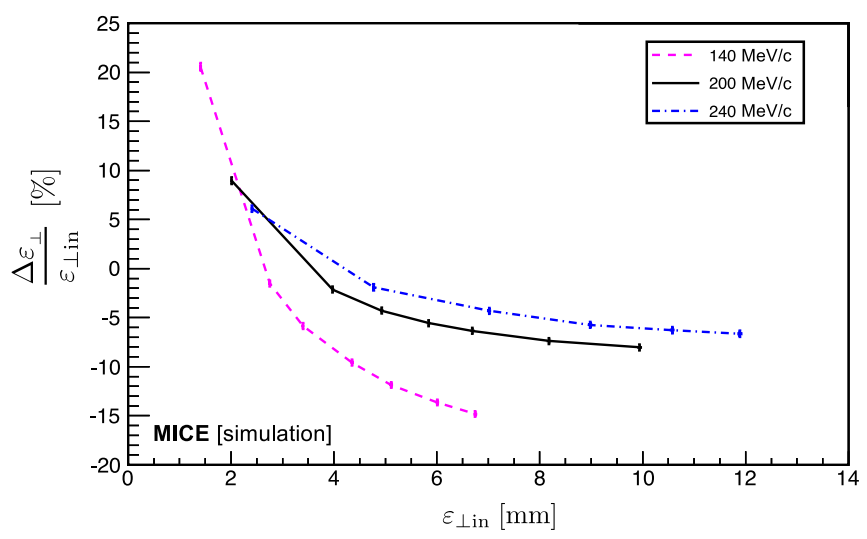

FIG. 10. Fractional change in emittance versus initial emittance $\left(\varepsilon_{\perp \text { in }}\right)$ for the cooling-demonstration lattice design measured at the tracker reference planes. The fractional change in emittance of the $140 \mathrm{MeV} / c, 200 \mathrm{MeV} / c$, and $240 \mathrm{MeV} / c$ lattices are shown as the purple-dashed, solid black, and dot-dashed blue lines, respectively. The error bars indicate the statistical precision that would be achieved using a sample of 100,000 muons.
$200 \mathrm{MeV} / c$ and $10 \mathrm{~mm}$ respectively, was $\sim 10 \%$. Figures 9 and 10 show the statistical uncertainties that will result from the reconstruction of a sample of 100,000 muons [42] with the configuration proposed in this paper. The instrumentation was specified to ensure that no single source of systematic uncertainty would contribute more than one third of the statistical uncertainty on the fractional change in emittance [15]. All of the instrumentation has been commissioned on the beam-line and performs to specification. The emittance-change evolution presented in Fig. 10 can therefore be measured with high significance.

\section{CONCLUSION}

An experiment by which to demonstrate ionization cooling has been described that is predicted by simulations to exhibit cooling over a range of momentum. The demonstration is performed using lithium-hydride absorbers and with acceleration provided by two $201 \mathrm{MHz}$ cavities. The equipment necessary to mount the experiment is either in hand (the superconducting magnets and instrumentation), or at an advanced stage of preparation. The configuration of the demonstration of ionization cooling has been shown to deliver the performance required for the detailed study of the ionization-cooling technique.

The demonstration of ionization cooling is essential to the future development of muon-based facilities that would provide the intense, well characterized low-emittance muon beams required to elucidate the physics of flavor at a neutrino factory or to deliver multi-TeV lepton-antilepton collisions at a muon collider. The successful completion of the MICE programme would therefore herald the establishment of a new technique for particle physics.

\section{ACKNOWLEDGMENTS}

The work described here was made possible by grants from the Science and Technology Facilities Council (UK), the Department of Energy and National Science Foundation (USA), the Instituto Nazionale di Fisica Nucleare (Italy), the Bulgarian Academy of Sciences, the Chinese Academy of Sciences, the Dutch National Science Foundation, the Ministry of Education, Science and Technological Development of the Republic of Serbia, the European Community under the European Commission Framework Programme 7 (AIDA project, Grant Agreement No. 262025, TIARA project, Grant Agreement No. 261905, and EuCARD), the Japan Society for the Promotion of Science and the Swiss National Science Foundation in the framework of the SCOPES programme. We gratefully acknowledge all sources of support. We are grateful to the support given to us by the staff of the STFC Rutherford Appleton and Daresbury Laboratories. We acknowledge the use of Grid computing resources deployed and operated by GridPP in the UK, http:// www.gridpp.ac.uk/. 
[1] S. Geer, Neutrino beams from muon storage rings: Characteristics and physics potential, Phys. Rev. D 57, 6989 (1998).

[2] M. Apollonio et al., Oscillation Physics with a Neutrino Factory, arXiv:hep-ph/0210192.

[3] D. V. Neuffer and R. B. Palmer, A high-energy highluminosity $\mu^{+}-\mu^{-}$collider in Proceedings of the Fourth European Particle Accelerator Conference EPAC 94, London, England (World Scientific, River Edge, NJ, 1994), p. 52.

[4] R. B. Palmer, Muon colliders, Rev. Accel. Sci. Techol. 07, 137 (2014).

[5] S. Y. Lee, Accelerator Physics, 3rd ed., edited by S. Y. Lee (World Scientific, Singapore, 2012).

[6] S. Schröder et al., First laser cooling of relativistic ions in a storage ring, Phys. Rev. Lett. 64, 2901 (1990).

[7] J. S. Hangst, M. Kristensen, J. S. Nielsen, O. Poulsen, J. P. Schiffer, and P. Shi, Laser cooling of a stored ion beam to 1 mK, Phys. Rev. Lett. 67, 1238 (1991).

[8] P. J. Channell, Laser cooling of heavy ion beams, J. Appl. Phys. 52, 3791 (1981).

[9] J. Marriner, Stochastic cooling overview, Nucl. Instrum. Methods Phys. Res., Sect. A 532, 11 (2004).

[10] V. V. Parkhomchuk and A. N. Skrinsky, Electron cooling: 35 years of development, Phys. Usp. 43, 433 (2000).

[11] A. N. Skrinsky and V. V. Parkhomchuk, Cooling methods for beams of charged particles, Fiz. Elem. Chastits At. Yadra 12, 557 (1981) [Sov. J. Part. Nucl. 12, 223 (1981)].

[12] D. Neuffer, Principles and applications of muon cooling, in Proceedings, 12th International Conference on HighEnergy Accelerators, HEACC 1983: Fermilab, Batavia, 1983, Vol. C830811 (Fermi National Accelerator Laboratory, Batavia, 1983), p. 481-484.

[13] D. Neuffer, Principles and applications of muon cooling, Part. Accel. 14, 75 (1983).

[14] D. Rajaram and V. C. Palladino, The status of MICE Step IV, in Proceedings, 6th International Particle Accelerator Conference (IPAC 2015): Richmond, Virginia, USA, 2015 (JACoW, Geneva, 2015), p. 4000.

[15] MICE Collaboration, MICE: An International Muon Ionization Cooling Experiment, http://mice.iit.edu/micenotes/ public/pdf/MICE0021/MICE0021.pdf (2003), MICE Note 21.

[16] M. Apollonio et al. (ISS Accelerator Working Group Collaboration), Accelerator design concept for future neutrino facilities, J. Instrum. 4, P07001 (2009).

[17] C. M. Ankenbrandt et al., Status of muon collider research and development and future plans, Phys. Rev. ST Accel. Beams 2, 081001 (1999).

[18] M. M. Alsharoa et al. (Neutrino Factory and Muon Collider Collaboration), Recent progress in neutrino factory and muon collider research within the Muon collaboration, Phys. Rev. ST Accel. Beams 6, 081001 (2003).

[19] R. B. Palmer, J. S. Berg, R. C. Fernow, J. C. Gallardo, H. G. Kirk, Y. Alexahin, D. Neuffer, S. A. Kahn, and D. Summers, A complete scheme of ionization cooling for a muon collider, in Proceedings of the 22nd Particle Accelerator Conference, PAC-2007, Albuquerque, NM (IEEE, New York, 2007), p. 3193.
[20] G. Penn and J. S. Wurtele, Beam Envelope Equations for Cooling of Muons in Solenoid Fields, Phys. Rev. Lett. 85, 764 (2000).

[21] C. Rogers, Ph.D. dissertation, Imperial College of London, 2008.

[22] D. Stratakis and R. B. Palmer, Rectilinear six-dimensional ionization cooling channel for a muon collider: A theoretical and numerical study, Phys. Rev. ST Accel. Beams 18, 031003 (2015).

[23] D. Neuffer, H. Sayed, T. Hart, and D. Summers, Final Cooling for a High-Energy High-Luminosity Lepton Collider, arXiv:1612.08960.

[24] A. V. Tollestrup and J. Monroe, Fermi National Accelerator Laboratory Technical Report No. FERMILAB-MUCOOL176, 2000.

[25] C. N. Booth et al., The design, construction and performance of the MICE target, J. Instrum. 8, P03006 (2013).

[26] C. N. Booth et al., The design and performance of an improved target for MICE, J. Instrum. 11, P05006 (2016).

[27] M. Bogomilov et al. (MICE Collaboration), The MICE Muon Beam on ISIS and the beam-line instrumentation of the Muon Ionization Cooling Experiment, J. Instrum. 7, P05009 (2012).

[28] D. Adams et al. (MICE Collaboration), Characterisation of the muon beams for the Muon Ionisation Cooling Experiment, Eur. Phys. J. C 73, 2582 (2013).

[29] R. Bertoni et al., The design and commissioning of the MICE upstream time-of-flight system, Nucl. Instrum. Methods Phys. Res., Sect. A 615, 14 (2010).

[30] R. Bertoni, M. Bonesini, A. de Bari, G. Cecchet, Y. Karadzhov, and R. Mazza, The construction of the MICE TOF2 detector, http://mice.iit.edu/micenotes/public/pdf/ MICE0286/MICE0286.pdf (2010).

[31] L. Cremaldi, D. A. Sanders, P. Sonnek, D. J. Summers, and J. Reidy, Jr., A Cherenkov radiation detector with high density aerogels, IEEE Trans. Nucl. Sci. 56, 1475 (2009).

[32] M. Ellis et al., The design, construction and performance of the MICE scintillating fibre trackers, Nucl. Instrum. Methods Phys. Res., Sect. A 659, 136 (2011).

[33] F. Ambrosino et al., Calibration and performances of the KLOE calorimeter, Nucl. Instrum. Methods Phys. Res., Sect. A 598, 239 (2009).

[34] R. Asfandiyarov et al., The design and construction of the MICE Electron-Muon Ranger, J. Instrum. 11, T10007 (2016).

[35] M. Leonova et al., MICE cavity installation and commissioning/operation at MTA, in Proceedings, 6th International Particle Accelerator Conference (IPAC 2015): Richmond, Virginia, USA, 2015 (JACoW, Geneva, 2015), p. 3342-3344.

[36] Y. Torun et al., Final commissioning of the MICE RF module prototype with production couplers, in Proceedings, 7th International Particle Accelerator Conference (IPAC 2016): Busan, Korea, 2016 (JACoW, Geneva, 2016), p. 474.

[37] R. C. Fernow and R. B. Palmer, Solenoidal ionization cooling lattices, Phys. Rev. ST Accel. Beams 10, 064001 (2007).

[38] C. D. Tunnell and C. T. Rogers, MAUS: MICE Analysis User Software, in Proceedings of the 2nd International Particle Accelerator Conference, San Sebastiáán, Spain (EPS-AG, Spain, 2011), p. 850. 
[39] S. Agostinelli et al. (GEANT4 Collaboration), GEANT4: A simulation toolkit, Nucl. Instrum. Methods Phys. Res., Sect. A 506, 250 (2003).

[40] J. Allison et al., Geant4 developments and applications, IEEE Trans. Nucl. Sci. 53, 270 (2006).
[41] R. Brun and F. Rademakers, ROOT: An object oriented data analysis framework, Nucl. Instrum. Methods Phys. Res., Sect. A 389, 81 (1997).

[42] C. Hunt, Ph.D. dissertation, Imperial College of London, 2017. 